\title{
Outcome of patients undergoing open heart surgery at the Uganda heart institute, Mulago hospital complex
}

Twalib O Aliku ${ }^{1,2}$, Sulaiman Lubega ${ }^{2}$, Peter Lwabi², Michael Oketcho², John O Omagino², Tom Mwambu²

1. Department of Paediatrics, Gulu University.

2. Uganda Heart Institute, Mulago Hospital Complex

\section{Abstract}

Background: Heart disease is a disabling condition and necessary surgical intervention is often lacking in many developing countries. Training of the superspecialties abroad is largely limited to observation with little or no opportunity for hands on experience. An approach in which open heart surgeries are conducted locally by visiting teams enabling skills transfer to the local team and helps build to build capacity has been adopted at the Uganda Heart Institute (UHI).

Objectives: We reviewed the progress of open heart surgery at the UHI and evaluated the postoperative outcomes and challenges faced in conducting open heart surgery in a developing country.

Methods: Medical records of patients undergoing open heart surgery at the UHI from October 2007 to June 2012 were reviewed.

Results: A total of 124 patients underwent open heart surgery during the study period. The commonest conditions were: venticular septal defects (VSDs) 34.7\% (43/124), Atrial septal defects (ASDs) 34.7\% (43/124) and tetralogy of fallot (TOF) in 10.5\% (13/124). Non governmental organizations (NGOs) funded $96.8 \%(120 / 124)$ of the operations, and in only patients (3.2\%) families pid for the surgeries. There was increaing complexity in cases operated upon from predominnty ASD (3.2\%) VS at dered 19 patients $(15.3 \%)$. Postoperative morbidity was low with arrhythmias, left ventricular dysfunction and re-operations being the commonest seen. Post operative sepsis occurred in only 2 cases $(1.6 \%)$. The overall mortality rate was $3.2 \%$

Conclusion: Open heart surgery though expensive is feasible in a developing country. With increased direct funding from governments and local charities to support open heart surgeries, more cardiac patients access surgical treatment locally. Keywords: Open heart surgery, Uganda Heart Institute

DOI: http://dx.doi.org/10.4314/ahs.v14i4.25

\section{Background:}

Heart disease is a disabling condition. Performing open heart surgery for congenital heart disease in resource limited countries is a major developmental challenge ${ }^{1}$ and in several sub Saharan countries this is often unavailable. As a result children in developing countries continue to have a significantly higher incidence and prevalence of serious congenital heart disease, partly due to lack of early corrective surgery ${ }^{2}$. Lack of facilities for pediatric cardiac surgery results in a large number of potentially preventable death and suffering ${ }^{3}$.The main options available to children with potentially correctable congenital heart defects include either referral abroad or having foreign surgeons come for short visits

\section{Corresponding author:}

Twalib Aliku,

Gulu University,

Department Of Paediatrics,

P.O Box 166 Gulu.

Email: aliku90@yahoo.com.

Mobile +256712551204 to operate on them ${ }^{1}$. Only few families can afford the referral expenses abroad, yet most charities that sponsor referrals abroad commonly take on those with good prognosis.

Increasingly, a few nongovernmental organization NGOs) prefer to sponsor operations of children in their countries of origin where facilities allow. This is not only cheaper but it also helps develop local skills to carry on the surgeries on their own. Eventually developing a specialized cardiac treatment centre in a resource limited setting through such skills transfer programs would be ideal 1 . An approach in which open heart surgeries are conducted locally by visiting teams alongside skills transfer to build local capacity has been adopted at the Uganda Heart Institute (UHI) since 2007.

The UHI is the national referral centre for treatment of cardiovascular diseases based at the Mulago Hospital complex. Closed heart surgeries like patient ductus arteniosus (PDA) ligation and pericardiectomy have been routinely performed at the UHI since 1997 . Open heart surgery at the UHI began in 2007 with support from visiting teams comprising cardiologists, a cardiac surgeon, perfusionists, cardiac intensive care unit (ICU nurses, cardiac anesthesiologists, fellows, biomedical personnel, and other staff from sponsoring NGOs. During these open heart surgery camps, the visiting teams carry a number of sundries for use in the operations. Preoperative surgical conferences were held, and the different cadres of visiting health personnel would work with their Ugandan counterparts enabling appropriate skills transfer.

Methods

The objective of the study was to evaluate the progress of open heart surgery at the UHI, to describe the postoperative outcomes and challenges faced in conducting these surgeries. This was a retrospective chart review where medical records of all patients undergoing open heart surgery for either congenital or acquired heart disease at the UHI from October 2007 to June 2012 were included. Those undergoing closed heart surgeries were excluded. Data including age, sex, cardiac diagnosis, type of operation, postoperative complications and ICU and hospital stay and funding source for the sur-

\begin{tabular}{|c|c|c|c|c|c|c|c|c|c|}
\hline \multirow[t]{2}{*}{ Age group } & \multicolumn{5}{|c|}{$\begin{array}{l}\text { Numbers of patients with Congenital } \\
\text { Heart disease }\end{array}$} & \multicolumn{3}{|c|}{$\begin{array}{l}\text { Numbers of patients } \\
\text { with Acquired Heart } \\
\text { Disease }\end{array}$} & \multirow[t]{2}{*}{ Total } \\
\hline & ASD & VSD & TOF & AS & OTHERS & RHD & EMF & Others & \\
\hline $\begin{array}{l}\text { Less than } 1 \\
\text { year }\end{array}$ & 0 & 0 & 0 & 0 & $2^{\#}$ & 0 & 0 & 0 & 2 \\
\hline $1-4$ years & 10 & 27 & 8 & 2 & $2^{\# \#}$ & 0 & 0 & 0 & 49 \\
\hline 5-11 years & 13 & 8 & 5 & 1 & $3^{*}$ & 1 & 0 & 0 & 31 \\
\hline $12-18$ years & 9 & 6 & 0 & 3 & $3 * *$ & 0 & 2 & $1 * * *$ & 24 \\
\hline Above 18 years & 11 & 2 & 0 & 1 & $2^{\# \# \#}$ & 2 & 0 & 0 & 18 \\
\hline Totals & 43 & 43 & 13 & 7 & 12 & 3 & 2 & 1 & 124 \\
\hline
\end{tabular}

Results

A total of 124 patients underwent open heart surgery during the study period. Of these, 66 (53.2\%) were male. Indications for surgery varied depending on the specific pathologies. Patients with congestive heart failure, large atrial septal defects (ASDs) or VSD (ventricular septal defects), those with severe valvular lesions, or those where surgery is the definitive therapy were operated.

The mean age of the patients was 9.39 years (SD9.76), with age range from 3 months to 52 years. The vast majority of patients were children (age less than 18 years), making up $93.5 \%(116 / 124)$ of the patients (see table

\section{Table 1: Distribution of different cardiac pathologies operated on by age groups}


The oldest patient with a VSD operated was a 34 year and severe left pulmonary artery (LPA) stenosis who cluded by the tricuspid valve presenting with heart fail- old with dysplastic pulmonary valves and two patients ure. A 12 year old girl with RHD having severe aortic year old with partial AV canal defect *** and A 19 year regurgitation underwent a Ross procedure.

old with an atrial myxoma.

\# Only surgeries in infancy included Re-implantation Only 2 children were operated before their first birthof an anomalous right pulmonary artery (RPA) arising day (a 3 month old patient with anomalous right pulfrom the ascending aorta back to the main pulmonary monary artery arising from the aorta who underwent artery (MPA) and severe pulmonary hypertension and and a 9 month old with tricuspid atresia who underwent a 9 month old who underwent bidirectional Glenn for a bidirectional Glenn procedure), with the age group tricuspid atresia.\#\#; These included an 18 month old 1-4 years making the majority followed by those aged to thrive and congestive heart failure, and a 2 year old operated between $1-4$ years of age; all Tetralogy of Falgirl with supracardiac Total anomalous pulmonary ve- lot patients were between 1-11 years and with all panous connection.\#\#\#; A 20 year old with partial atrio tients with ASDs nearly equally distributed in the other venticular (AV) Canal defect *included a 5 year old who age groups after infancy. Congenital heart disease surunderwent a bidirectional Glenn for Tricuspid atresia, geries made up $96 \%(119 / 124)$ of the procedures. The a 10 year old with Noonan's syndrome who underwent commonest conditions were ventricular septal defects surgical valvuloplasty for severe pulmonary stenosis (VSD) in 34.7\% (44/124), atrial septal defects (ASDs) with dysplastic valves, and a 5 year old with Large PDA in 34.7\% (43/124) and TOF in 10.5\% (13/124), see figure 1.

Figure 1: Types of congenital heart defects operated at the Uganda Heart institute. Other operation included Valve replacement surgery in 3 patients with RHD, Bidirectional Glenn for tricuspid atresia in two children, TAPVR repair, Aortopulmonary window repair, Pulmonary valve stenosis, Atrioventricular canal defects. Patients with aortic stenosis were those with subaortic membrane resection only.

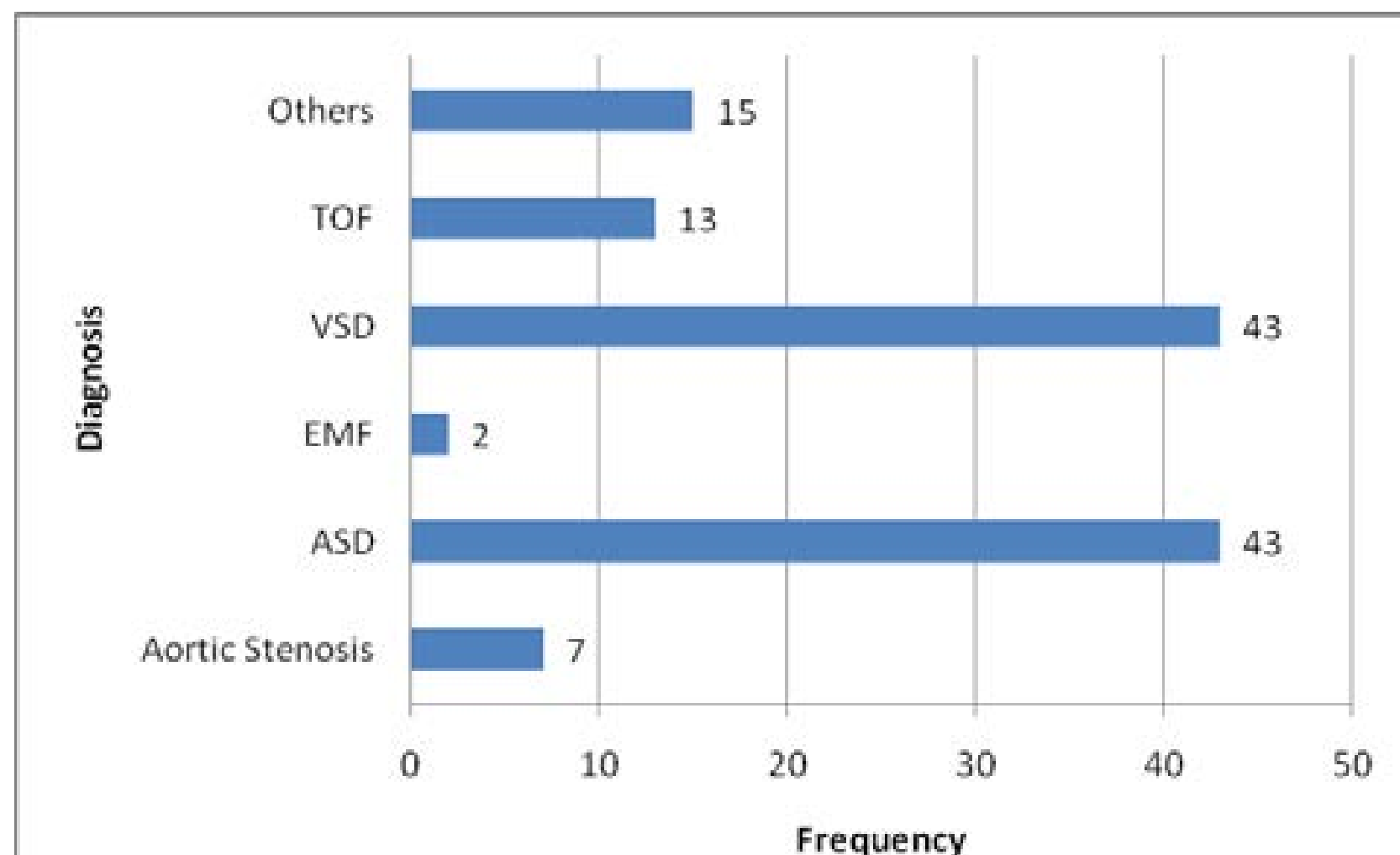

Acquired heart diseases operated included 3 patients with rheumatic heart disease, 2 patients with endomyocardial fibrosis (EMF) and an adolescent with atrial myxoma. The youngest patient with acquired heart disease was a 12 year old with rheumatic severe aortic regurgitation who underwent a Ross procedure. There was increasing complexity in cases operated from predominantly ASDs and VSDs at the beginning to more complex cases like TOFs and Total anomalous pulmonary venous drainage from 2010. Foreign charities funded $82.3 \%(102 / 124)$ of the operations, and only 4 patients $(3.2 \%)$ paid for their surgeries. A local NGO funded the rest.

The local team independently operated 19 patients $(15.3 \%)$. The local team started independently operating ASDs in 2009 and then moved on to the VSDs. The vast majority of patients independently operated by the local team were secundum ASDs (13/19), followed by $3(10.6 \%)$ patients being the commonest complication, Table 2 Showing postoperative complications.

\begin{tabular}{|l|l|} 
Post operative complications & Number $/ 124(\%)$ \\
\hline Need for re-intervention & $6(4.9)$ \\
\hline Postoperative arrhythmias* & $13(10.6)$ \\
\hline Left Ventricular Dysfunction & $6(4.9)$ \\
\hline Pericardial Effusion & $6(4.9)$ \\
\hline Postoperative Bleeding & $4(3.3)$ \\
\hline $\begin{array}{l}\text { Sildernafil use for pulmonary } \\
\text { hypertension }\end{array}$ & $3(2.4)$ \\
\hline 30- Day mortality & $4(3.3)$ \\
\hline
\end{tabular}

Note ${ }^{*}$ These excluded sinus tachycardia. Arrhyth- valvular pulmonary stenosis, a 17 year old with EMF, a mias seen included atrial fibrillation, Junctional three year old patient with Large VSD and Large PDA, tachycardia,complete AV block. One patient went on and a 15 year old with subaortic stenosis ), retrosterto have a permanent pacemaker inserted. 3 patients nal abscess evacuation in a 12 year old girl with subneeded a temporary pacemaker; two had amiodarone to pumonic VSD and a 4 year old with malaligned VSD control their arrhythmias. Most patients with pericar- with complete heart block who underwent permanent dial effusions had small effusions except a patient with pacemaker (VVI) implantation.

EMF who had a moderate pericardial effusion that was drained.

Post operative sepsis (defined as wound sepsis or intrathoracic abscess or pyrexia persisting for more than Re-inteverntions included re-exploration due to exces- 48 hours postoperatively with leukocytosis or pyrexia
sive hemorrhage from chest drains in 4 patients (a ten
with continuation of antibiotics after chest tube drain year old patient having Noonan's syndrome with severe removal) occurred in only 2 cases $(1.6 \%)$. These includ- 
ed the patient with retrosternal abscess and an 8 year The overall mortality was $3.2 \%(4 / 124)$. The earliest old with perimembranous VSD who had sternotomy post operative death was six hours after the initial sursite sepsis. gery, with the latest being 40 days after the surgery (see figure 2)

Figure 2: Kaplan - Meier survival analysis showing the time to death among study patients.

\section{Survival Function}

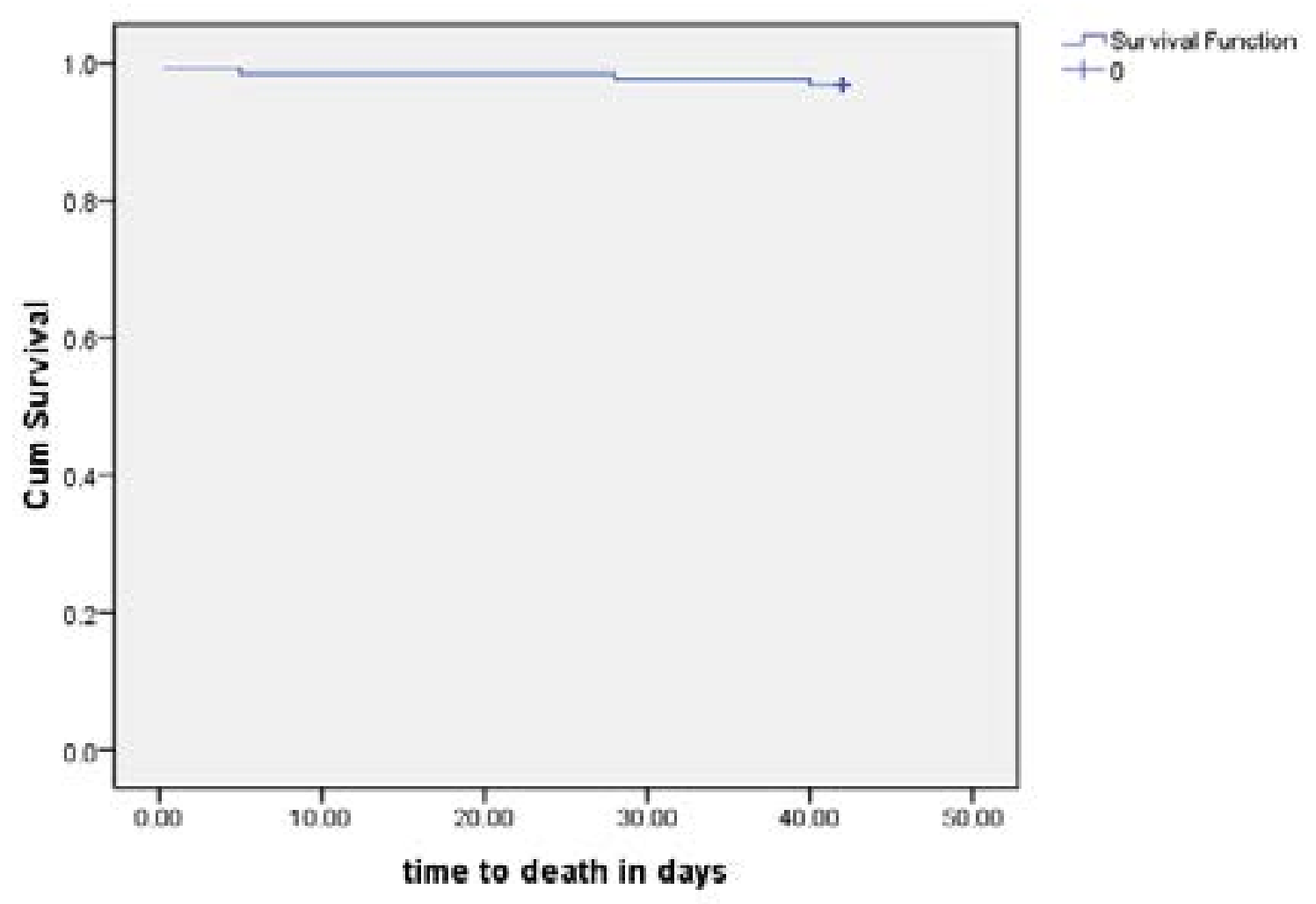

Those who died included two adolescent patients with plasma and vitamin K. She remained ventilator dependEMF having multiorgan failure, a 2 year old child with ent from initial surgery. Three days later she developed a VSD and a large PDA, and a 15 year old patient with hepatic dysfunction with elevation of liver enzymes, a large Inlet VSD with severe pulmonary hypertension worsening jaundice and had continued hypotension who developed severe left ventricular dysfunction. f of the four who died were above 14 years. All of those tive day from multiorgan failure.

who died had at least one post operative complication.

One of the EMF patients was a 17 year old girl with The other EMF patient was a 15 year old boy with Right biventricular EMF having severe tricuspid regurgitation Ventricular EMF having severe Tricuspid regurgitation. and uncontrolled right heart failure who underwent He underwent resection of fibrotic RV endocardium, excision of fibrotic RV endocardium, with tricuspid tricuspid annuloplasty and bidirectional Glenn. He reannuloplasty and bidirectional Glenn procedure. She quired inotropic support for three days after surgery, developed low cardiac output with hypotension im- and was weaned off the ventilator in 3 days. He spent 5 mediately after surgery that required inotropic support. days in the ICU and was transferred to the main ward. Eight hours after surgery she had excessive chest tube A week later he developed marked respiratory distress, bleeding that required re-exploration where bleeders with large bilateral pleural effusions and moderate periwere identified and ligated. She received fresh frozen cardial effusion that was drained. He was readmitted to the ICU and required mechanical respiratory. He subsequently developed hepatic dysfunction, recurrent pleural effusions, coma, remained on pressor support to maintain cardiac output and developed renal dysfunction. He died on day postoperative day 42 from multiple organ failure.

The two year old with a large perimembranous VSD and large PDA underwent VSD closure and PDA ligation due to poor weight gain and congestive heart failure despite optimal medical therapy. Two hours postoperative the patient developed increased bleeding from chest drainage and bleeding from the sternotomy wounds that necessitated re-operation.

It was found that accidentally the descending aorta wa ligated instead of the large PDA that was of identical size. End to end anastomosis of the descending aorta was done. The child died from acute kidney failure 6 hours from initial operation. The 15 year old with a large inlet VSD with cleft mitral valve (with moderate regurgitation) had surgery due to severe pulmonary hypertension that was deemed reversible clinically. Postoperatively he developed moderate LV systolic dysfunction that improved on inotropic support for 48 hours, and was given sildernafil for severe pulmonary hypertension. He spent 5 days in the ICU and was subsequently discharged from hospital after two weeks. Four weeks after initial surgery he was readmitted with symptoms of worsening congestive heart failure. His repeat echo showed severe left ventricular dysfunction and he died from ventricular tachycardia.

\section{Discussion}

The experience at the Uganda heart institute shows that open heart surgery is feasible in the setting of a developing country. Most of our patients are operated beyond the age of one year. Currently our pediatric open heart surgery service is severely constrained in meeting the needs of neonates and infants. This situation is common in sub-Saharan countries'. In a study of 51 patients undergoing open heart surgery at Lagos State University Teaching hospital, the mean age was $29 \pm 15$.6years 5 .

With appropriate care, up to $85 \%$ of children diagnosed with congenital heart disease can reach adulthood ${ }^{6}$. A
great obstacle to the provision of appropriate pediatric cardiac services remains a lack of appropriately trained medical personnel ${ }^{4}$ and funding for the surgeries.

Major public health issues such as the HIV/AIDS pandemic, coupled with a myriad of tropical diseases like
B and malaria ravage resource limited countries. This makes congenital or acquired cardiovascular diseases less of a priority in government resource allocation ${ }^{7}$. As such direct government spending towards corrective heart surgery is often lacking or severely limited. To compound the problem further, the prohibitive cost of open heart surgery is virtually out of reach of the vast majority of families in developing countries who have children with heart disease. This was clearly demonstrated in the present study where only 4 patients were able to afford surgery out of pocket. As a result, many patients with surgically correctable heart disease are left with no option but to continue medial therapy and suffer recurrent complications needing outpatient care and hospital admissions. This is the main reason for the low number of patients operated independently by the local team.

This trend of events in the long term amount to higher costs compared to the cost of a single staged corrective surgical repair ${ }^{4}$. The open heart surgery operations in many developing countries still rely considerably on funding from foreign organizations, as seen in this study and elsewhere ${ }^{5}$

The scarcity of a in-country funding sources, unavailability of some specialized sundries within developing countries along with staffing constraints still limit the number of patients in need of open heart surgery that could be operated locally by the resident open heart surgery teams.

Increasing patient volumes in cardiac surgery is es sential in boosting confidence of operating teams and refining critical skills that would enhance the development of centers for cardiac surgery and help to improve outcomes. ${ }^{8}$. Access to needed cardiac surgery can be increased if locally based private foundations directly contribute to costs of open heart surgery pro$\mathrm{gram}^{7}$ together with greater direct government funding. This would help minimize the increased morbidity and mortality associated with older age at operation as was seen in this study. Another possible source for direct funding cardiac surgery programs directly engaging locally based corporate entities and civil society in charity funding?.

The mortality rates of 3.3\% observed here in the present study are comparable to those seen elsewhere with cardiac centres and other database that are reported to range from $1.8-6.1 \%{ }^{10-13}$. Edwin et al reported mortality rates of 3\% among patients with congenital heart disease undergoing open heart surgery, with a reduction in

African Health Sciences Vol 14 Issue 4, December 2014 
mortality with increase in the volumes of patients operated $^{8}$. The low postoperative morbidity encountered in this study could be attributable to rigorous patient selection. Late presentation to hospital and less than optimal preoperative evaluation may be a major contributor to postoperative mortality in resource poor settings? Currently we do not have a pediatric intensivist. Pediatric cardiac intensive care services that specialize in the care of cardiac patients needing postoperative management is an essential part of paediatric cardiac services and contributes significantly to outcomes ${ }^{6}$. However the patients operated at the UHI were significantly older, there were no neonatal surgeries, and involved significantly less complex congenital heart disease which could have contributed to lower rates of complications. Neonatal surgeries or surgeries for very complex conpostoperative morbidity and mortality ${ }^{10-11,13}$.

The visiting teams also had cardiac intensivists that could have helped reduce the mortality in the present study. Starting with relatively less complex- low risk congenital heart disease surgery with rigorous preoperative assessment is essential to prevent high morbidity and mortality in starting open heart surgery programs in a developing country. The low rate for sepsis seen here compared to other centers in sub Saharan Africa ${ }^{5}$ could be attributable to rigorous sepsis control measures and the local practice of keeping on antibiotics longer till all chest drains are removed (typically day 2 postoperative), absence s of non emergent open heart surgery (OHS) cases as many patients are prepared electively.

Sample size was attained by enrolling all patients who underwent OHS during the period. We recognize that this study may not be powered enough to draw specific conclusions, but serves to describe experiences at the UHI during the study period.

\section{Conclusion}

The experience at the UHI has shown that conducting open heart surgery operations at local sites positively impacts on the ability of local cardiac teams to have hands on training, exposes most local health cadres to

Okafor, Ugochukwu, Azike Jerome An audit of inhas enabled the cardiac team at the UHI to start per- tensive care unit admission in a pediatric cardiothoracic forming more open heart surgeries of increasing com- population in Enugu, Nigeria. Pan African Medical Jourplexity independently. Lack of direct funding for open nal. 2010;6:10

heart surgery programs is a major obstacle limiting the 8. Edwin, F, Tettey M, Aniteye E, Sereboe L, Tama-

number of children that can be operated by local teams tey M, Entsua-Mensah K, Kotei, D B-GK. The develas only very few families can pay for the costs of the

\section{References}

1. Noedir, A. G Stolf. Congenital heart surgery in a developing country: a few men for a great challenge. ;16:1874-5

(n) improving paediatric heart http:www.childrens heartlink.org.

3. Magdi, H Yaccoub. Establishing pediatric cardiovas4. Hoosen, EGM, Cilliers AM, Hugo-Hamman CT, Brown SC, Harrisberg JR, Takawira FF, Govendragelo G, Lawrenson J, Hewitson J. Optimal paediatric cardiac services in South Africa-what do we need? Statemen of the paediatric cardiac society of south Africa. SA Heart. 2012;7(1):10-6 a work in progress. Journal of Cardiothoracic Surgery 2013

Warnes, Liberthson R, Danielson GK et al. Tas Force 1 . The changing profile of congenital heart disease in adult life. J AM Coll Cardiol 2001; 37(5):1170-5 Task Force 1. The changing profile of congenital hear disease in adult life. J AM Coll Cardiol. 2001;37(5):1170opment of cardiac surgery in West Africa-the case of Ghana. Pan Afr Med J 2011;9:15.
9. Christine, Awuor Yuko-Jowi. African experiences of Stratifications at a Tertiary Care Center in Thailand. 9umanitarian cardiovascular medicine: a Kenyan petspective. Cardiovasc Diagn Ther. 2012;2(3):231-9. 10. Rakhi, Balachandran, Suresh G Nair, Sunil S Go- leider R. M. Comparison of pediatric cardiac surgical palraj, Balu Vaidyanathan, and R Krishna Kumar. Dedi- mortality ratesfrom national administrative data to concated pediatric cardiac intensive care unit in a develop- temporary clinical standards. Annals of Thoracic Suring country: Does it improve the outcome? Ann Pediatr gery 2009;87(1):216-23.

Cardiol. 2011;4(2):122-6.

13. Rohde, S. L, Matebele M, Pohlner P, Radford D, 11. Chodchanok, Vijarnsorn, Duangmanee Laohapra- Wall D, and Fraser J.F. Excellent cardiac surgical outsitiporn, Kritvikrom Durongpisitkul, et al. Surveillance comes in paediatric indigenous patients, but follow-up of Pediatric Cardiac Surgical Outcome Using Risk difficulties. Heart Lung and Circulation 2010;19(9):517- 